\title{
Genomic insights from whole genome sequencing of four clonal outbreak Campylobacter jejuni assessed within the global C. jejuni population
}

Clifford G. Clark ${ }^{1 *}$, Chrystal Berry ${ }^{1}$, Matthew Walker ${ }^{1}$, Aaron Petkau², Dillon O. R. Barker ${ }^{3,4}$, Cai Guan ${ }^{1}$, Aleisha Reimer ${ }^{1}$ and Eduardo N. Taboada ${ }^{3,4}$

\begin{abstract}
Background: Whole genome sequencing (WGS) is useful for determining clusters of human cases, investigating outbreaks, and defining the population genetics of bacteria. It also provides information about other aspects of bacterial biology, including classical typing results, virulence, and adaptive strategies of the organism. Cell culture invasion and protein expression patterns of four related multilocus sequence type 21 (ST21) C. jejuni isolates from a significant Canadian water-borne outbreak were previously associated with the presence of a CJIE1 prophage. Whole genome sequencing was used to examine the genetic diversity among these isolates and confirm that previous observations could be attributed to differential prophage carriage. Moreover, we sought to determine the presence of genome sequences that could be used as surrogate markers to delineate outbreak-associated isolates.

Results: Differential carriage of the CJIE1 prophage was identified as the major genetic difference among the four outbreak isolates. High quality single-nucleotide variant (hqSNV) and core genome multilocus sequence typing (cgMLST) clustered these isolates within expanded datasets consisting of additional C. jejuni strains. The number and location of homopolymeric tract regions was identical in all four outbreak isolates but differed from all other $C$. jejuni examined. Comparative genomics and PCR amplification enabled the identification of large chromosomal inversions of approximately $93 \mathrm{~kb}$ and $388 \mathrm{~kb}$ within the outbreak isolates associated with transducer-like proteins containing long nucleotide repeat sequences. The $93-\mathrm{kb}$ inversion was characteristic of the outbreak-associated isolates, and the gene content of this inverted region displayed high synteny with the reference strain.

Conclusions: The four outbreak isolates were clonally derived and differed mainly in the presence of the CJIE1 prophage, validating earlier findings linking the prophage to phenotypic differences in virulence assays and protein expression. The identification of large, genetically syntenous chromosomal inversions in the genomes of outbreakassociated isolates provided a unique method for discriminating outbreak isolates from the background population. Transducer-like proteins appear to be associated with the chromosomal inversions. CgMLST and hqSNV analysis also effectively delineated the outbreak isolates within the larger C. jejuni population structure.
\end{abstract}

Keywords: Campylobacter, Whole genome sequence, Phylogenetic analysis, Core genome MLST, Genomic inversions, Chemotaxis proteins, Transducer-like proteins

\footnotetext{
* Correspondence: clifford.clark@phac-aspc.gc.ca

${ }^{1}$ Division of Enteric Diseases, National Microbiology Laboratory, Public Health

Agency of Canada, Canadian Science Centre for Human and Animal Health,

1015 Arlington Street, Winnipeg, MB R3E 3R2, Canada

Full list of author information is available at the end of the article
} 


\section{Background}

Campylobacter jejuni is the predominant bacterial species causing human enteric disease globally [1-5]. In the United States the reported number of human clinical cases is second only to Salmonella, and the incidence of disease attributed to C. jejuni is increasing in many countries [2]. This organism can be recovered from many animals, including cattle, pigs, chickens, wild birds, flies, and protozoa, from retail foods, especially chicken, and from the environment $[4,6,7]$. Despite the observation that many animals may serve as hosts for $C$. jejuni, poultry is thought to be the predominant source of human infections [4].

Surveillance for $C$. jejuni has been accomplished via DNA fingerprinting or molecular typing methods of variable discriminatory power, including pulsed-field gel electrophoresis (PFGE), multilocus sequence typing (MLST), ribosomal MLST (rMLST), flagellin short variable region (flaSVR) sequencing, and porA gene (major outer membrane protein, MOMP) sequencing [8, 9]. Using these methods, C. jejuni outbreak detection occurs relatively infrequently compared to detection of outbreaks caused by other enteric bacteria [10]. Water, milk, and chicken products are the most frequently reported sources of $C$. jejuni outbreaks $[4,10]$, with the majority of human cases assumed to be sporadic. Much of what we know about the passage of bacterial pathogens through the food chain and their interaction with human populations comes from characterization of foodborne-outbreak events [10]. The use of newer technologies and higher resolution methods like nextgeneration whole genome sequencing (WGS) provides more robust outbreak detection and characterization. In addition, comparative genomic methods can be used to investigate the biological and pathogenic mechanisms contributing to bacterial interactions with their environment, including their propagation and survival strategies as bacteria navigate the food chain to cause human clinical illness.

WGS is rapidly becoming a primary analytic method for bacterial phylogenetic studies, detection of bacterial pathogens in clinical laboratories, and outbreak detection and analysis [11-14]. However, in specific instances it may be more economically efficient to perform WGS on a smaller number of isolates representative of a larger population, such as when initiating studies aimed at the development of high-throughput, low-cost molecular subtyping assays for rapid or large-scale screening [9, 15]. Phylogenetic studies based on high-quality single nucleotide variant (SNV) distances and overall gene content have to date supported the current understanding that $C$. jejuni comprises a highly diverse group of organisms $[3,16]$. Furthermore, WGS has been used to characterize isolates associated with milk- and water-borne $C$. jejuni outbreaks in Finland [17, 18] and to retrospectively identify case clusters from groups of isolates that were initially presumed to be associated with sporadic infections [19]. Genomic methods such as ribosomal MLST (rMLST), core genome MLST (cgMLST), and whole genome MLST (wgMLST) are complementary methods to extant, pregenomic era typing schemes. Each of these newer genomic methods provides incrementally greater resolution, discriminatory power, and insight into $C$. jejuni populations while retaining the capability of accurately detecting case clusters [20]. However, the utility of WGS is not limited to phylogenetic analysis, and can provide clues about the virulence of an organism, its niche adaptation, its chromosomal structure, and other aspects of its biology [21-24]. We were therefore interested in undertaking a comprehensive analysis of isolates associated with the largest recorded Canadian Campylobacter outbreak, reasoning that valuable insights into the Campylobacter genome could be obtained through a robust analysis of whole genome sequences.

Four closely related $C$. jejuni isolates from a significant Canadian waterborne outbreak in 2000 [25] were subjected to WGS. These isolates were epidemiologically linked to the outbreak, previously typed as Walkerton outbreak strain 1 [26], and had common HS (heat-stable) and HL (heat labile) serotypes, biotype, MLST sequence type, and fla-SVR type. The PFGE restriction patterns varied according to the presence and location of the CJIE1 prophage within the bacterial chromosome [27]. Our previous investigations into the role of CJIE1 indicated that carriage of this prophage is linked to differences in bacterial phenotype [28] and protein expression [29]. Three isolates carrying the CJIE1 prophage exhibited increased adherence and invasion in cell culture compared with an isolate lacking the prophage [28]. Furthermore, there were differences in protein expression levels in the three isolates carrying the prophage compared with the isolate lacking the prophage [29]. These results suggested the CJIE1 prophage may affect diverse aspects of the biology of the organism. Previous, unpublished DNA-microarray data suggested that the isolates were genetically very similar except for the carriage of the CJIE1 prophage. However, it remained unknown if there were additional genetic differences other than those revealed using the earlier typing methods and DNA microarray technology.

Our first goal was to investigate the total genetic content among the isolates to determine if the isolates were 1) clonal, 2) differed mainly by the presence or absence of the CJIE1 prophage, and 3) harboured genetic differences with potential biological relevance other than the CJIE1 prophage. A second goal was to determine whether a thorough comparative genome analysis would provide additional insight into the population structure, genome plasticity, and virulence potential of the outbreak isolates. We determined that the genomic location and number of 
homopolymeric tracts within the four isolates and several other strains with complete, finished WGS suggested that the location and number of homopolymeric tracts may be useful to inform strain identity, relatedness, or clonal descent when assessing bacterial isolates that appear to be clonally or epidemiologically related. Comparative analysis also revealed the presence of a large, genetically syntenous chromosomal inversion, indicating structural heterogeneity of the chromosome and suggesting that the inversion may either have a biologically relevant phenotype or could be used as a marker specific for the outbreak isolates. Together, these results demonstrate the ability of WGS-based analysis to provide a great deal of in-depth, disparate, yet highly-valuable information about the organism under study.

\section{Results}

The major genomic difference among outbreak isolates is the presence of CJIE1 prophage

Draft genomes of the four $C$. jejuni outbreak-associated isolates were obtained by paired-end sequencing on an Illumina MiSeq platform. Complete, closed, and finished genomes were obtained using a combination of read mapping to a reference genome, NCTC11168 = ATCC 700819, and bridging of contig gaps by Sanger sequencing (Fig. 1). The genome sizes of isolates $00-2425(1,718,982$ bases), 00-2538 (1,719,369 bases), and 00-2544 (1,719,532 bases) are very similar, differing by a maximum of 550 bases. In contrast, the genome size of isolate 00-2426 was $1,680,813$ bases, a difference of between 38,169 and 38,719 bases from the previous three genomes. This difference is very close to the estimated size of prophage CJIE1 in $C$. jejuni isolate $00-2425$ of approximately 38,000 bases. Both isolates $00-2425$ and $00-2538$ were annotated as having 1744 CDSs, compared with 1792 CDSs for isolate 00-2544 and 1686 CDSs for isolate 00-2426. DNA sequence alignments revealed that the four isolates were genetically syntenic with nearly identical gene content in all four strains apart from the CJIE1 prophage (Figs. 1 and 2), the presence of four tRNA loci (tRNA-Asp, tRNA-Val, tRNA-Glu, tRNA-Lys) near the aas gene only in isolate 00-2426 (data not shown), and the different chromosomal insertion site of CJIE1 in isolate 00-2544 (Fig. 1). This latter observation validated an earlier observation demonstrating chromosomal heterogeneity of the CJIE1 prophage in an otherwise clonal population of isolates [27].

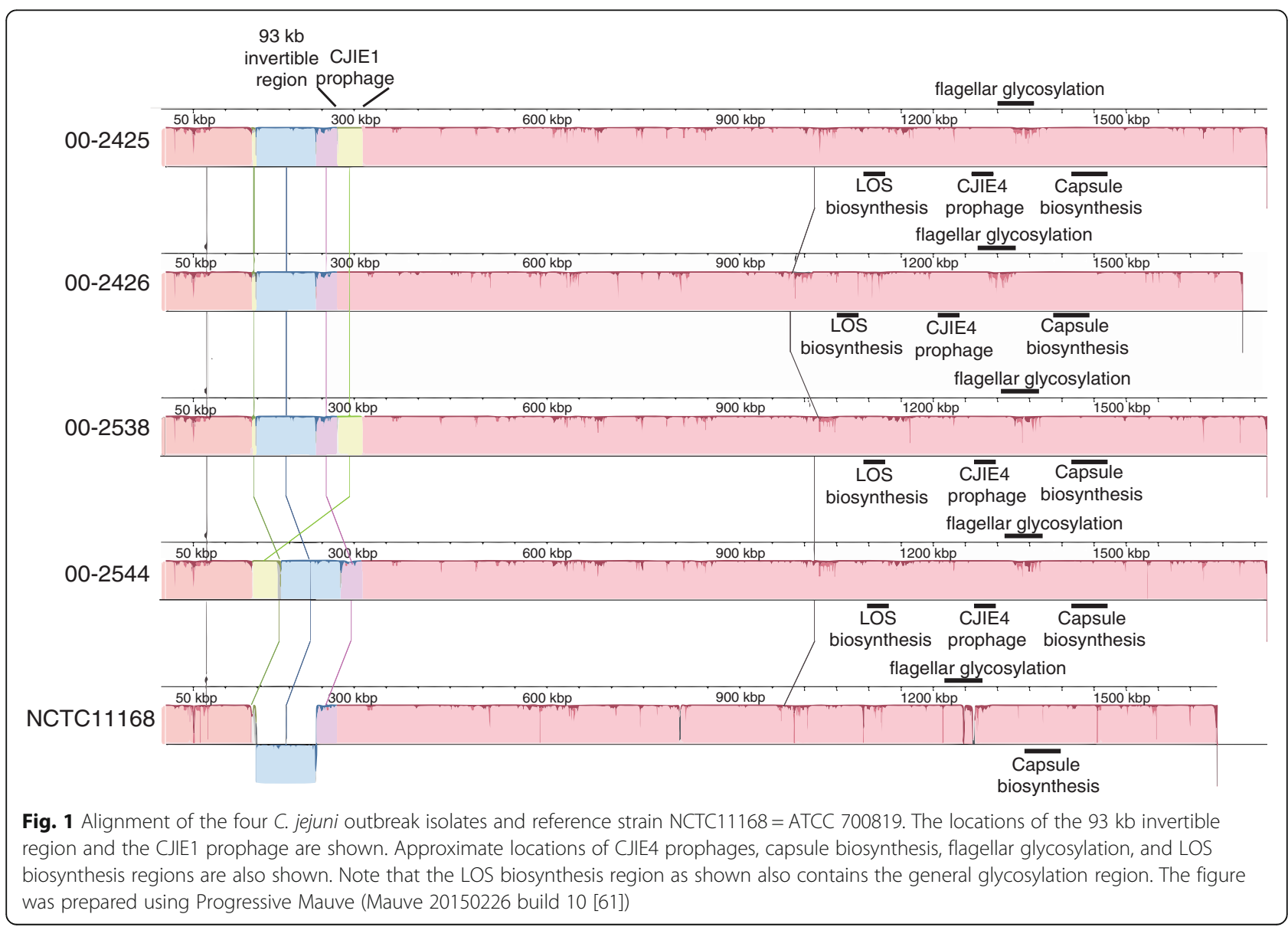




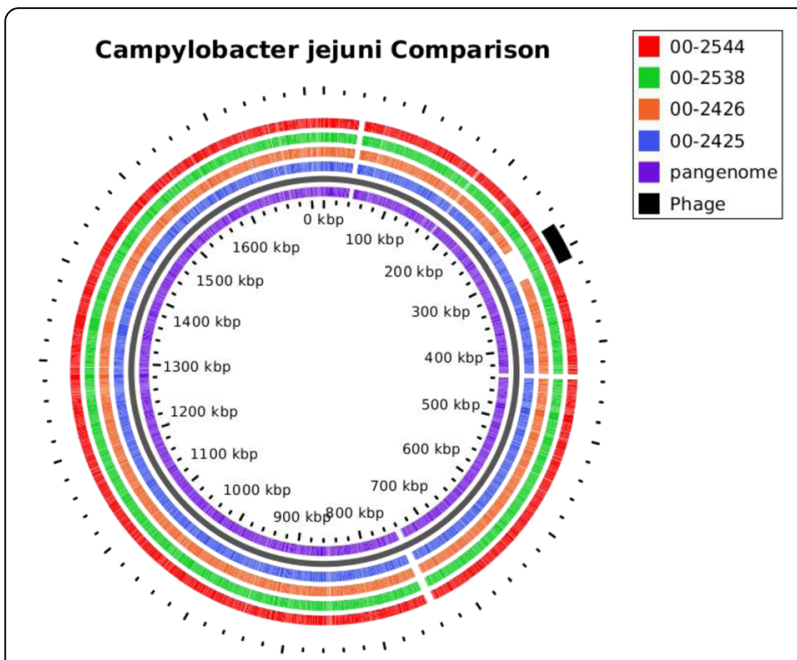

Fig. 2 Blast atlas of whole genome sequences of $C$. jejuni isolates 00-2425, 00-2426, 00-2538, and 00-2544. The Blast atlas was prepared using GView Server [56]

Whole genome sequencing also revealed a $46,902 \mathrm{bp}$ plasmid harboured by one isolate, C. jejuni 00-2544, showing high homology to pTet plasmids in the NCBI repository but varying due to the insertion of a transposable element comprised of the IS605 transposase (OrfB family) and integrase-resolvase (OrfA) genes adjacent to a gene encoding aminoglycoside 3'-phosphotransferase a short distance away from the plasmid-encoded tet $(\mathrm{O})$.

\section{Phylogenetic analysis indicates that the four outbreak isolates are clonally related}

High quality SNVs (hqSNVs; Table 1) were identified and interrogated as outlined in the Methods section. Whole genome sequence alignments of the outbreak isolates to the reference strain, NCTC11168 $=$ ATCC 700819, were performed. NCTC11168 = ATCC 700819 was selected as the reference for hqSNV analysis based on a Neighbor-Joining analysis using 31 publicly-available complete $C$. jejuni genomes that revealed this strain was a very close genetic neighbour to the outbreak isolates (Additional file 1: Figure S1). The core phylogenetic analysis revealed that, overall, the outbreak isolates differed by a total of $15 \mathrm{SNVs}$ from one another, and in both the core genome and whole genome phylogenetic trees the outbreak isolates formed a distinct group within the overall topology of C. jejuni isolates (Fig. 3, Additional file 1: Figure S1). SNV analysis indicated they were most closely related to other MLST clonal complex (CC) ST-21 strains, especially YH001, which is sequence type (ST) 806, and the two NCTC11168 strains, which are ST43.

A hqSNV at position $1,035,018$ in isolate $00-2426$ (NCTC11168 position 1,031,608 in Table 1) results in the locus encoding the threonine/serine transporter SstT becoming a pseudogene. As discussed earlier [29] the
NCTC11168 annotation and available data indicates that this protein is a serine transporter without any additional function and, as such, would not be expected to impart global regulatory effects. Of the $15 \mathrm{hqSNVs}$ detected (Table 1), 13 were present only in isolate $00-2544$ and two were found only in isolate 00-2426. In addition to the SNVs identified using the hqSNV core genome analysis, whole genome sequence using NUCmer (see Methods, [30, 31]) revealed several additional SNVs (Additional file 2: Table S1). Many of these were unique to isolate $00-2544$, and were located in two genes encoding motility functions; flagellin A and the motility accessory factor protein homologous to Cj1341c. We hypothesize that these are allelic differences arising from homologous recombination. These data provide further evidence that isolate $00-2544$ is not as closely related as the other three outbreak isolates. The analysis also revealed a SNV at position 682,886 in isolate 00-2425 (position 644,332 in isolate 00-2426) that would result in the phosphate acetyltransferase protein product being a pseudogene in isolate $00-2426$. However, this position was located within a homopolymeric tract of $\mathrm{T}$ residues. Homopolymeric tracts are intrinsically repetitive and are therefore omitted from SNV analysis. The full-length phosphate acetyltransferase protein may be expressed due to rapid changes caused by homopolymeric tract length variability.

We further subjected the four isolates to cgMLST analysis using a schema based on 732 core genes (unpublished data). Because the four outbreak isolates belong to ST21, we included additional genomes belonging to this sequence type obtained from the Bacterial Isolate Genome Sequence Database (BIGSdb; $n=181$ ) [32] or sequenced by our group $(n=9)$ to provide greater context to the hqSNV results. The cgMLST results (Fig. 4) supported our phylogenetic findings and confirmed that the outbreak isolates were genetically highly similar. Core genome analysis revealed that ST21 represents a heterogeneous population of isolates comprised of distinct clusters with limited intra-cluster variability and high levels of inter-cluster variability (data not shown). Whereas the average number of variant loci between pairs of genomes was 172.1, the four outbreak isolates clustered with four additional Canadian isolates with an average of 9.39 variant loci. Within this cluster, isolates 00-2425, 00-2426 and 00-2538 showed the least amount of genetic variation, differing at an average of 2.0 loci. With an average of 5.6 variant loci, isolate 00-2444 showed slightly higher divergence with respect to the three other outbreak isolates. Four additional $C$. jejuni isolates were in the same immediate cluster as the Walkerton outbreak isolates 00-2425, 00-2426, 00-2538, and 00-2544 (Fig. 4). Three were isolated in 2007 from Ontario, while the fourth was 
Table 1 High quality core SNVs in outbreak isolates using NCTC11168= ATCCC 700819 as the reference

\begin{tabular}{|c|c|c|c|c|c|c|c|}
\hline Position in NCTC11168 & NCTC 11168 & $00-2425$ & $00-2426$ & 00-2538 & $00-2544$ & Protein & Locus in NCTC11168 \\
\hline 192795 & G & A & A & A & G & amidophosphoribosyltransferase & Cj0196c \\
\hline 226072 & C & A & A & A & C & intergenic & \\
\hline 231489 & G & A & A & A & G & MFS transport protein & Cj0250c \\
\hline 492104 & T & T & T & T & C & periplasmic protein & Cj0530 \\
\hline 524447 & C & C & T & C & C & periplasmic protein & Cj0561c \\
\hline 937334 & C & C & C & C & T & MtaB & Cj1006c \\
\hline 959966 & G & G & G & G & A & DNA gyrase subunit A & Cj1027c \\
\hline 1031608 & T & T & G & T & T & serine/threonine transporter SstT & Cj1097 \\
\hline 1137283 & C & C & C & C & T & intergenic & \\
\hline 1173179 & T & T & T & T & C & hypothetical protein & Cj1245c \\
\hline 1181839 & G & A & A & A & G & LPS assembly protein & Cj1252 \\
\hline 1189659 & A & A & A & A & G & major outer membrane protein & Cj1259 \\
\hline 1298000 & A & A & A & A & G & secreted serine protease & Cj1365c \\
\hline 1515973 & A & A & A & A & C & D-lactate dehydrogenase & Cj1585c \\
\hline 1630514 & C & C & C & C & T & 2-isopropylmalate synthase & Cj1719c \\
\hline
\end{tabular}

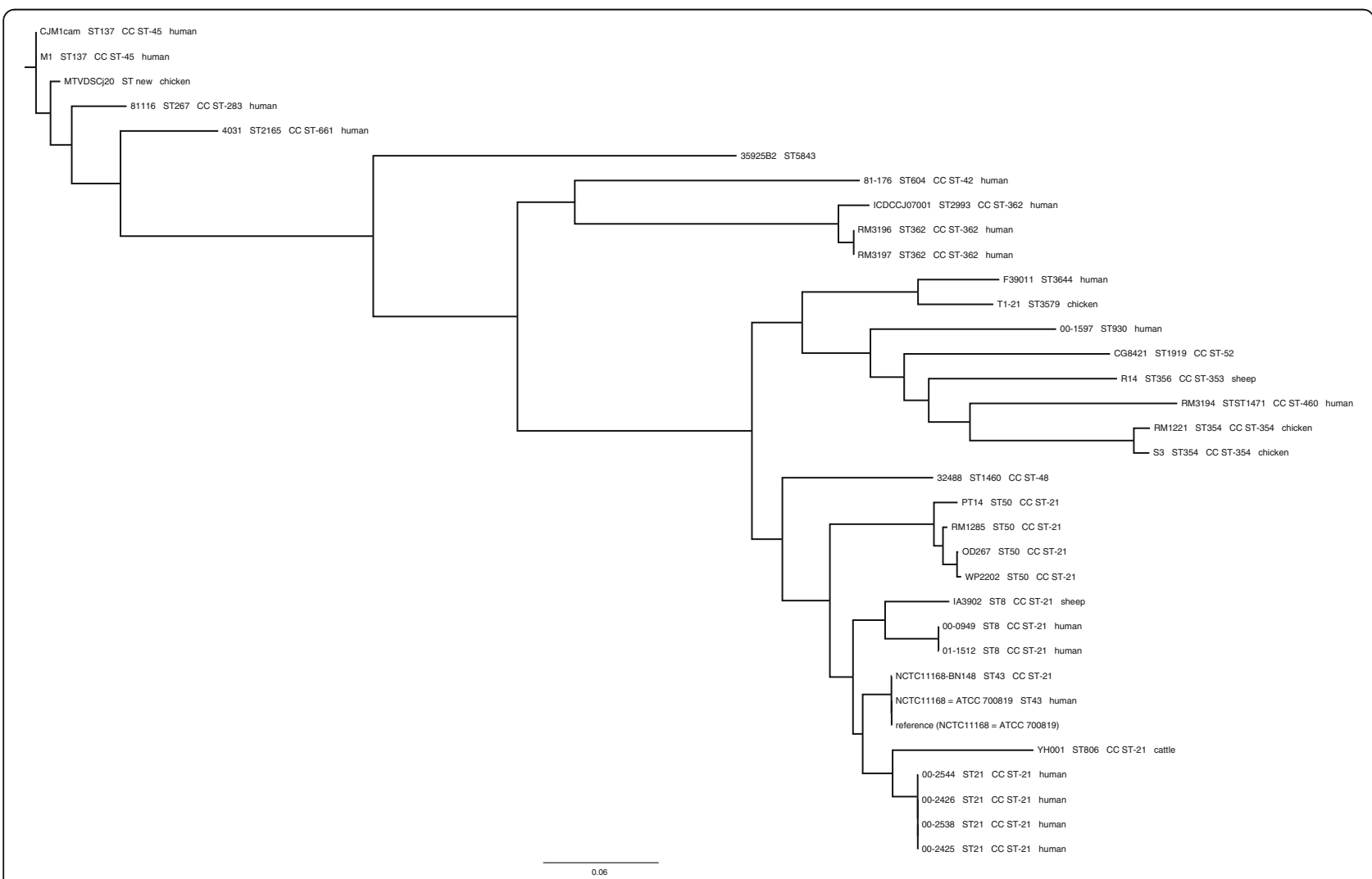

Fig. 3 Phylogenetic tree of C. jejuni sequenced in this study compared with publicly available C. jejuni. The tree was constructed using hqSNV analysis as outlined in the Methods section 


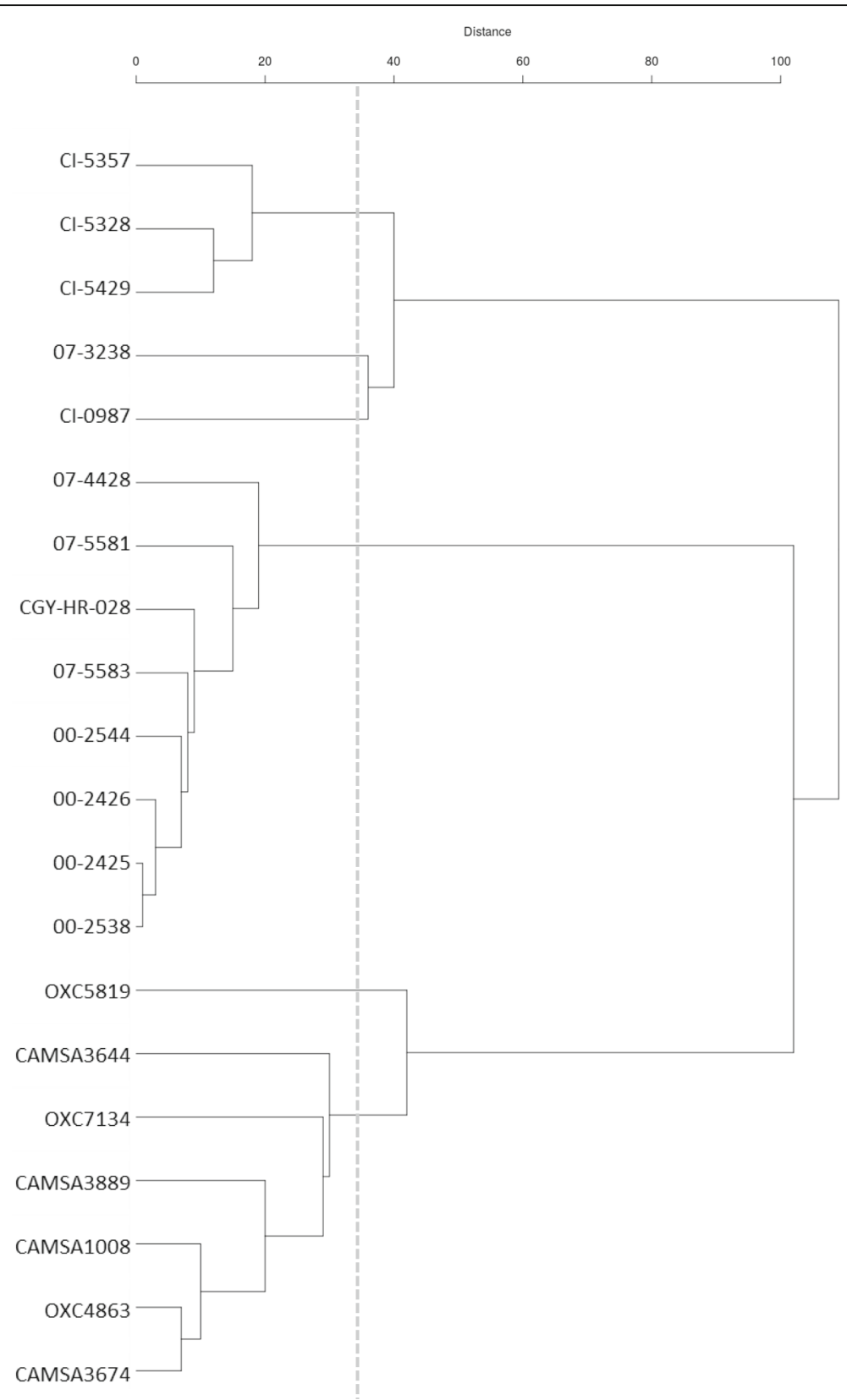

Fig. 4 cgMLST tree showing phylogenetic relationships of the four outbreak isolates compared with other ST21 isolates. The tree was created using isolates within one of 21 clades (Clade 12) arising from a larger analysis of ST21 strains (unpublished data). Clade 12 contains C. jejuni strains most closely related to the four isolates (00-2425, 00-2426, 00-2538, 00-2544) sequenced for this work

isolated in 2006 from Alberta, indicating that this clade was both stable in time and geographically widespread. This group of eight strains was part of a larger cluster, Clade 12, which in turn comprised only a very small branch on a dendrogram of a ST21 phylogeny with a total of 21 clades (unpublished data). In this context the four Walkerton isolates are very closely related to each other and likely to be clonally derived, consistent with epidemiological data from the outbreak.

\section{Homopolymeric tracts are useful for assessing relationships among isolates}

Because the number and location of homopolymeric tracts is known to vary among different $C$. jejuni isolates [33], we identified the locations of polyG/polyC tracts more than seven bases long in the four isolates sequenced for this study by searching DNA sequences in GenBank files for strings of Gs or Cs of eight nucleotides or longer and associating the position with the location of genes (Table 2). 
Table 2 Consensus homopolymeric tracts in genomes of the four sequenced $C$ jejuni isolates

\begin{tabular}{|c|c|c|c|c|}
\hline \multirow[t]{2}{*}{ Homolog in NCTC11168 } & \multicolumn{4}{|c|}{ Length of homopolymeric tract } \\
\hline & $00-2425$ & $00-2426$ & $00-2538$ & 00-2544 \\
\hline 115 bp upstream of L-asparaginase start codon (Cj0029) & $10 \mathrm{C}$ & $10 \mathrm{C}$ & $10 \mathrm{C}$ & $10 \mathrm{C}$ \\
\hline Cj0045c iron-binding protein & $10 \mathrm{C}$ & $10 \mathrm{C}$ & $10 \mathrm{C}$ & $10 \mathrm{C}$ \\
\hline Cj0170 methyltransferase & $8 \mathrm{C}$ & $10 \mathrm{C}$ & $9 \mathrm{C}$ & $10 \mathrm{C}$ \\
\hline Between Cj0564 and Cj0565 & $10 \mathrm{G}$ & $11 \mathrm{G}$ & $11 \mathrm{G}$ & $10 \mathrm{G}$ \\
\hline Cj0617 carbonic anhydrase & $9 \mathrm{G}$ & $10 \mathrm{G}$ & $10 \mathrm{G}$ & $9 \mathrm{G}$ \\
\hline Cj0628 lipoprotein & $9 \mathrm{G}$ & $9 \mathrm{G}$ & $9 \mathrm{G}$ & $9 \mathrm{G}$ \\
\hline Cj0676 kdpA potassium-transporting ATPase A subunit & $10 \mathrm{G}$ & $9 \mathrm{G}$ & $10 \mathrm{G}$ & $8 \mathrm{G}$ \\
\hline Cj0685c invasion protein CipA & $9 \mathrm{G}$ & $8 \mathrm{G}$ & $9 \mathrm{G}$ & $9 \mathrm{G}$ \\
\hline Between Cj0742 (membrane protein) and 16S rRNA locus & $10 \mathrm{C}$ & $10 \mathrm{C}$ & $10 \mathrm{C}$ & $10 \mathrm{C}$ \\
\hline Cj1051c cjel restriction modification enzyme & $9 \mathrm{C}$ & $10 \mathrm{C}$ & $9 \mathrm{C}$ & $9 \mathrm{C}$ \\
\hline Cj1139c wlaN $\beta$-1,3-galactosyltransferase & $8 \mathrm{C}$ & $8 \mathrm{C}$ & $8 \mathrm{C}$ & $9 \mathrm{C}$ \\
\hline Cj1 145 hypothetical protein & $8 \mathrm{C}$ & $10 \mathrm{C}$ & $9 C$ & $9 \mathrm{C}$ \\
\hline Cj1295 aminopeptidase & $9 \mathrm{G}$ & $9 \mathrm{G}$ & $8 \mathrm{G}$ & $10 \mathrm{G}$ \\
\hline Cj1296 AAC(3) family N-acetyltransferase & $9 \mathrm{G}$ & $9 \mathrm{G}$ & $9 \mathrm{G}$ & $9 \mathrm{G}$ \\
\hline Cj1305c carbonic anhydrase & $10 \mathrm{C}$ & $9 \mathrm{C}$ & $10 \mathrm{C}$ & $11 \mathrm{C}$ \\
\hline Cj1306c carbonic anhydrase & $9 \mathrm{C}$ & $9 \mathrm{C}$ & $9 \mathrm{C}$ & $9 \mathrm{C}$ \\
\hline Cj1310 hypothetical protein & $9 \mathrm{C}$ & $9 \mathrm{C}$ & $9 \mathrm{C}$ & $10 \mathrm{C}$ \\
\hline Cj1321 promoter region; 37 bp upstream of Cj1321 start & $10 \mathrm{G}$ & $10 \mathrm{G}$ & $11 \mathrm{G}$ & $11 \mathrm{G}$ \\
\hline Cj1325 methyltransferase & $10 \mathrm{G}$ & $10 \mathrm{G}$ & $11 \mathrm{G}$ & $10 \mathrm{G}$ \\
\hline Cj1335-1336 motility accessory factor, maf4 & $9 \mathrm{G}$ & $10 \mathrm{G}$ & $9 \mathrm{G}$ & $9 \mathrm{G}$ \\
\hline Cj1342 motility accessory factor, maf7 & $8 \mathrm{C}$ & $9 \mathrm{C}$ & $9 \mathrm{C}$ & $9 \mathrm{C}$ \\
\hline Cj1420c methyltransferase & $10 \mathrm{C}$ & $10 \mathrm{C}$ & $10 \mathrm{C}$ & $10 \mathrm{C}$ \\
\hline Cj1421c sugar transferase & $9 \mathrm{C}$ & $9 \mathrm{C}$ & $9 \mathrm{C}$ & $9 \mathrm{C}$ \\
\hline Cj1422 sugar transferase & $9 \mathrm{C}$ & $9 \mathrm{C}$ & $9 \mathrm{C}$ & $9 \mathrm{C}$ \\
\hline Cj1426c methyltransferase & $11 \mathrm{C}$ & $12 \mathrm{C}$ & $9 \mathrm{C}$ & $11 \mathrm{C}$ \\
\hline Cj1429 hypothetical protein & $11 \mathrm{C}$ & $8 \mathrm{C}$ & $10 \mathrm{C}$ & $11 \mathrm{C}$ \\
\hline Cj1437 hypothetical protein & $9 \mathrm{C}$ & $9 \mathrm{C}$ & $9 \mathrm{C}$ & $10 \mathrm{C}$ \\
\hline
\end{tabular}

Homopolymeric tract lengths corresponding to expression of the full length protein are in bold font

All four outbreak isolates carried 27 homopolymeric tracts in the same genomic locations, though the number of nucleotides within some of these exhibited apparent strain-specific variation. Consistent with previous reports [33], both the number and locations of homopolymeric tracts were different in the eight other strains included for comparisons (Additional file 3: Table S2), further supporting the premise that the four Walkerton C. jejuni are closely related clonal variants more similar to one another than to other $C$. jejuni strains. However, these four isolates carried 27 of the 29 homopolymeric tracts present in NCTC11168, missing homopolymeric tracts only in the type IIS restriction/modification enzyme (Cj0031) and the sodium:sulfate symporter (Table 2; Additional file 3: Table S2). In contrast, only 17/26 C. jejuni YH001 homopolymeric tracts were in the same location in C. jejuni YH001 and the four outbreak isolates. YH001 is an isolate from beef liver [34] that appeared to have substantial differences from the outbreak isolates and NCTC11168 (both HS:2) in the capsular polysaccharide biosynthesis region, which was most similar to regions encoding HS:4 (data not shown). Methods that do not take into account hypervariable regions may overestimate isolate relatedness. This was confirmed in the Neighbor-Joining analysis of $C$. jejuni complete genomes discussed in the previous section (Additional file 1: Figure S1).

Eight of the homopolymeric tracts present in the four Walkerton isolates were also present in a majority of the eight whole genome-sequenced strains analyzed (Additional file 3: Table S2). Their locations were within homologs of proteins annotated in NCTC11168 as Cj0045c (iron-binding protein/bacteriohemerythrin), Cj0617 (carbonic anhydrase), Cj0685c (invasion protein, CipA), Cj1295 
(aminopeptidase), Cj1310 (hypothetical protein), Cj1342 (maf7), between Cj0564 and Cj0565, or between Cj0742 and the $16 \mathrm{~S}$ rRNA locus.

\section{Identification and characterization of a previously unidentified large chromosomal inversion in outbreak isolates}

The process of genome closing and finishing enabled the identification of a large, genetically syntenous region present in the outbreak isolates that was in reverse orientation compared to the reference genome. This was revealed during gap closure using PCR primers consistent with the reported genome sequences of $C$. jejuni strains NCTC11168 and RM 1221, shown in Table 3. These primers were designed to bind outside repeat regions at the ends of the contigs that affected the assembly of the genome.

While use of the $\mathrm{Cj}-\mathrm{F} 1$ and $\mathrm{Cj}-\mathrm{R} 1$ or $\mathrm{Cj}-\mathrm{F} 2$ and $\mathrm{Cj}-\mathrm{R} 2$ primers pairs did not result in gap closure, $\mathrm{PCR}$ using the $\mathrm{Cj}-\mathrm{F} 1 / \mathrm{Cj}-\mathrm{F} 2$ and $\mathrm{Cj}-\mathrm{R} 1 / \mathrm{Cj} \mathrm{R} 2$ primer combinations (Table 3 ) successfully amplified a $\sim 2.5 \mathrm{~kb}$ product spanning the gaps between contigs. This indicates that within the genomes of the four isolates an approximately $93-\mathrm{kb}$ section of the genome between genes homologous to cj0143 (ABC transporter binding protein) and cj0263 (ZupT) was inverted relative to NCTC11168 (see Fig. 5, Additional file 4: Figure S2, Additional file 5: Table S3) and several other annotated $C$. jejuni genomes within the NCBI database (data not shown). The NCTC11168 strain (HS:2, ST43) used in our laboratory as a reference strain for PCR determination of inversion sequence termini was found to harbour an $\sim 92-\mathrm{kb}$ chromosomal inversion that was extremely similar to the four sequenced outbreak isolates. This experimental finding was in contrast to the sequence annotation for NCTC11168= ATCC 700819 in NCBI, which does not contain an annotated inversion. It is unclear if this was because our reference strain is a variant of NCTC11168 arising from repeated laboratory passage or if this is the result of an error with the annotated sequence in the NCBI repository. Analogs of the 93-kb inversion were not limited to HS:2 or ST21 isolates, though to date the inversions we have found all belong to CC ST-21 strains. An inversion with the same core gene content was detected in isolates 00-6200 (HS:4,13, ST806, CC ST-21; NCBI accession No. NZ_CP010307) and YH001 (HS:4, ST806, CC ST-21; NCBI accession No. NZ_CP010058.1).

The C. jejuni NCTC11168 original annotation (GenBank Accession No. AL111168) documents the existence of three large sequence repeats located in the loci cj0144, cj0262c, and cj1564, all of which were annotated as methyl-accepting chemotaxis proteins, later renamed transducer-like proteins (Tlps) [35]. Different Tlps have divergent $\mathrm{N}$-termini, with the repeats located in DNA sequences encoding the $\mathrm{C}$-termini. The repeats associated with cj0144 and cj0262 flank a region in NCTC11168 of 92,358 nt that shares 99\% nt identity with the 93,129-kb inverted region in the four outbreak isolates (Additional file 4: Figure S2; Additional file 5: Table S3). Genome analysis revealed that each of the four isolates sequenced for this study (00-2425, 002426, 00-2538, 00-2544) also harbour large sequence repeats within genes analogous to those in NCTC11168. These repeats were in proteins annotated as a methylaccepting chemotaxis protein (Tlp3), a ribose and galactose chemoreceptor protein (a novel Tlp, here designated Tlp12), and a methyl-accepting chemotaxis protein (a second copy of Tlp3); the first Tlp3 and Tlp12 flank the 93-kb invertible element in the outbreak isolates. The gene content within the invertible region may be maintained even though there may be changes in the adjacent methyl-accepting chemotaxis loci/Tlps. This warrants further investigation.

The repeat sequences associated with each of the three tlp loci displayed 100\% sequence identity in all four outbreak isolates, although the different tlp genes harbour repeats of different lengths (Additional file 6: Figure S3).

Table 3 Primers used to detect the 93- and 388-kb inversions and the pTet plasmid

\begin{tabular}{|c|c|c|c|}
\hline Primer target & Primer location & Primer name & Primer sequence $\left(5^{\prime}-3^{\prime}\right)$ \\
\hline Inversion (93kb) & cj0143c & $\mathrm{Cj}-\mathrm{F} 1$ & ATGCTTGAGGTGCTATACTGACAC \\
\hline Inversion (93kb) & cj0145 & $\mathrm{Cj}-\mathrm{R} 1$ & CCTTATCCTTAAGCATAGCAGCAC \\
\hline Inversion (93kb) & cj0261c & $\mathrm{Cj}-\mathrm{F} 2$ & ACCCCAGTTCCACATCCTATATC \\
\hline Inversion (93kb) & cj0263 (zupT) & $\mathrm{Cj}-\mathrm{R} 2$ & TGGTAAATTGGCAAACTCACAG \\
\hline Inversion (388 kb) & cj0261c & Cjlnv5F & ACCCCAGTTCCACATCC \\
\hline Inversion (388 kb) & cj1562-1563 & Cjlnv5R & AACCCATCGACTTCATTTG \\
\hline Plasmid & cpp50 & cpp50tetOF1 & GAACTITACTTGCACGGAATGGAG \\
\hline Plasmid & tetO & cpp50tetOR1 & GGCCTGGCGTATCTATAATGTTGA \\
\hline Plasmid & virB4 & virB4rcvapF1 & ATCTAGCTCATCATCATCTTCTGC \\
\hline Plasmid & $\operatorname{vapD}$ & virB4rcvapR1 & CTCGTCTITCATCTATTGGTTCTT \\
\hline
\end{tabular}




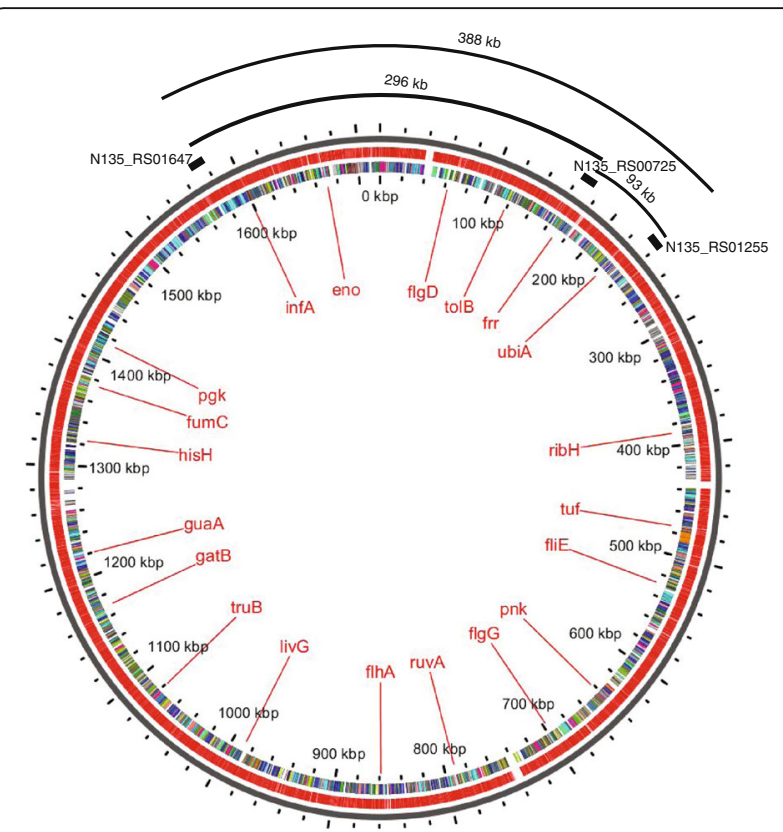

Fig. 5 Schematic diagram of the 00-2425 genome with repeats and potential inversions. The image was obtained with the 00-2425 GenBank file using GView Server [56] and shows the locations of the repeat sequences associated with chemotaxis genes in the 00-2425 genome and genomic regions that may be inverted by recombination between these repeats. The approximate locations of the sequence repeats (black boxes) and the approximate size of the fragments that might be inverted are shown. The inside multicolored circle shows Clusters of Orthologous groups, while the red circle represents the 00-2425 genome

In the outbreak isolates the first repeat (going clockwise from the origin of replication, Fig. 5) was 1979 nt, the second $1011 \mathrm{nt}$, and the third $1970 \mathrm{nt}$ (Additional file 6: Figure S3). We have designated the 1979 nt repeat unit "repeat 1", the $1101 \mathrm{nt}$ repeat unit "repeat 2" and third 1970 repeat unit "repeat 3". Repeat 2 is in reverse orientation to the other two in the outbreak isolates, and all three repeats display high levels of nucleotide identity over variable sequence lengths: 1969/1970 (99\%) between repeats 1 and 3; 1004/1011 (99\%) between repeats 1 and 2; 994/1004 (99\%) between repeats 2 and 3 (Additional file 7: Figure S4). The two tlp3 genes exhibit identity over 1955/1956 nt using NCBI blastn, and the respective proteins are identical over 650/651 aa when aligned using NCBI blastp. Repeats 1 and 3 are both larger than the $t l p 3$ genes with which they are associated. We hypothesize that the high percentage of nucleotide identity between repeats is sufficient to support recombination events leading to the large chromosomal rearrangements and subsequent invertible region.

The corresponding repeat 1 in NCTC11168, associated with the locus cj0144 encoding a Tlp2 protein, is $1079 \mathrm{nt}$; this repeat displays 99\% identity over 1079 nt with the first repeat in all four sequenced outbreak isolates. Repeat 2 in
NCTC11168, associated with locus $c j 0262 c$ encoding a Tlp4 protein, is 1147 nt in length, and has 1000/1013 (99\%) identity with the second repeat in the four outbreak isolates except for four indels. It is placed in reverse orientation relative to the first repeat in NCTC11168 similarly to the first two repeats in the four outbreak isolates. Finally, repeat 3 in NCTC11168, associated with locus cj1563c encoding Tlp3, is $1079 \mathrm{nt}$ compared with $1970 \mathrm{nt}$ for repeat 3 in the sequenced outbreak isolates. There is a 1951/1956 (99.7\%) nt identity between the NCTC11168 and outbreak-isolate $t l p 3$ genes. Studies are underway to investigate the diversity and extent of these repeat units within an expanded $C$. jejuni population and will be the subject of a separate report.

The maintenance of two sequence repeats flanking an invertible element may indicate that the element harbours genes subject to selective pressure(s). This hypothesis is supported by the observation that following an inversion event genes on the leading strand will now be switched to the lagging strand with respect to chromosomal replication, effectively changing the gene dosage [21, 35]. Inspection of the predicted protein identities for genes within the $93 \mathrm{~kb}$ invertible cassette revealed almost identical gene content, with very few exceptions (Additional file 4: Figure S2, Additional file 5: Table S3). BLAST searches using nucleotide sequences of the 92$\mathrm{kb}$ invertible region (including repeats) of NCTC11168 $=$ ATCC700819 as the query sequence and the 93-kb invertible region of isolate $00-2425$ as the subject sequence identified a region with of 92130/92207 (99\%) identity with 23/92207 gaps (0\%), an Expect value of 0.0, and a BLAST score of $1.659 \mathrm{e}+05$ bits (184022). Thus, despite the annotation differences, the gene/protein content of the two regions is highly similar. Included among the loci encoded in the invertible element are a number of membrane proteins, chemotaxis proteins, RNAmodifying enzymes, and a subset of iron acquisition proteins on the leading strand of the four $C$. jejuni isolates, some of which are proposed to have a functional role in environmental or niche adaptation. In contrast, the lagging strand (genes in complementary orientation) carried a large complement of transport proteins, including theTonB receptor and TonB transporter, ExbB and ExbD, among others.

Outbreak isolates are characterized by a 93-kb chromosomal inversion but demonstrate variable pTet plasmid carriage We were interested in determining whether the $93 \mathrm{~kb}$ inversion was a characteristic feature of isolates associated solely with the Walkerton outbreak. Not all isolates associated with the outbreak were previously typed by molecular typing methods or PFGE [26] though all were subjected to phage typing, HS typing, and HL typing [36]. Therefore, MALDI-TOF was used to verify the species 
(jejuni or coli) of selected isolates (data not shown), and MLST types were determined retrospectively for 95 additional $C$. jejuni isolates from the outbreak analysis. Isolates from humans or bovine manure were considered to be associated with the outbreak strains [26] if they were isolated during the outbreak investigation, considered epidemiologically outbreak-associated based on time and location, and belonged to CC ST-21. We were interested in identifying outbreak-strain markers for analysis of this particular outbreak or that could have been used to better characterize the Campylobacter population in that particular geographical area at that time.

PCR detection of the 93-kb inversion indicated that the inversion was a characteristic of outbreak strains since it was detected in $97 \%$ of the human isolates and $100 \%$ of the cattle isolates (Table 4), consistent with the clonal spread of the organism within the local cattle population and its subsequent introduction into humans through water [25]. The inversion was not prevalent in other CC ST-21 isolates tested, though the numbers were small (Table 4). The inversion was present at much lower frequencies in the relatively few non-Walkertonoutbreak isolates available and was detected in an isolate of strain NCTC11168 which has been utilized for years in our laboratory as a reference strain for different investigations. Together these results indicate that the 93-kb inversion was closely associated with the Walkerton $C$. jejuni isolates and could be used as a secondary, confirmatory trait of outbreak isolates. Although PCR detection of the inversion was highly effective and reliable as a tool in this instance, and could be rapidly used during an outbreak investigation, WGS-based analysis using the methods described in this paper were equally effective.

The discovery of a pTet plasmid homolog in a single outbreak isolate was accomplished using two sets of PCR primers (Table 3). Amplicons for both primer sets were detected in $15 \%$ of the human Walkerton CC ST21 isolates, increasing to $64 \%$ detection among Walkerton CC ST-21 isolates from cattle manure (Table 5). The plasmid was detected at higher frequencies in nonWalkerton CC ST-21 isolates from humans and animals, as well as in C. coli (not CC ST-21) isolates from animals. It appeared that either the human population was less frequently infected with plasmid-containing strains from bovine feces or that the plasmid was readily lost upon human infection. In a minority of cases only one of the two amplicons was detected, suggesting some heterogeneity in the plasmid content or nucleotide changes within the primer binding sites. The plasmid was not a reliable marker for outbreak strains and appears to have been rapidly lost in the human population after infection with strains of bovine origin through contaminated water.

Though data were limited, 7/10 of the non-Walkerton human CC ST-21 isolates with the complete pTet plasmid belonged to two PFGE types. Of these, five had PFGE patterns CASAI.0009-CAKNI.0032 and were from New Brunswick (2), Québec (2), and Ontario (1), while one each from Québec and New Brunswick had PFGE patterns CASAI.0026-CAKNI.0031. This suggests the pTet plasmid may have been circulating in a geographically delimited reservoir of related isolates.

\section{Identification of a $\mathbf{3 8 8} \mathrm{kb}$ inverted region}

We hypothesized that additional chromosomal inversions could have arisen in C. jejuni due to homologous recombination events mediated by the high nucleotide identity observed among the three repeat elements identified in the sequenced outbreak isolates (see Fig. 5). PCR analysis using primers designed to bridge the junctions of each of these potential inversions was performed on a subset of $C$. jejuni and C. coli isolates (see Methods, Strains and growth conditions), including isolates that did not harbour either the non-inverted sequence or the 93-kb inversion. Only one isolate (00-5949; HS:2, ST21, fla-SVR type 49) produced an $\sim 3 \mathrm{~kb}$ amplicon using the cjinv5 primer set designed to specifically detect an inversion between gene homologs of $c j 0261 c$ and $c j 1562-1563$

Table 4 Detection of a 93-kb inversion in isolates associated with the Walkerton outbreak investigation

\begin{tabular}{|c|c|c|c|c|}
\hline \multirow[t]{2}{*}{ Isolate characterization } & \multicolumn{4}{|c|}{ Detection of $93 \mathrm{~kb}$ inversion using PCR primers } \\
\hline & Total isolates & Non-inversion & Inversion & No PCR product \\
\hline Walkerton human, CC ST-21 & 110 & $3(3 \%)$ & 107 (97\%) & 0 \\
\hline Walkerton human, not CC ST-21 & 14 & $14(100 \%)$ & 0 & 0 \\
\hline Walkerton bovine, CC ST-21 & 22 & 0 & $22(100 \%)$ & 0 \\
\hline Walkerton bovine, not CC ST-21 & 20 & $15(75 \%)$ & $1(5 \%)$ & $4(20 \%)$ \\
\hline Non-Walkerton human, CC ST-21 & 10 & $8(80 \%)$ & $1(10 \%)$ & $1(10 \%)$ \\
\hline Non-Walkerton human, not CC ST-21 & 4 & $3(75 \%)$ & 0 & $1(25 \%)$ \\
\hline Non-Walkerton animal, CC ST-21 & 5 & $3(60 \%)$ & $1(20 \%)$ & $1(20 \%)$ \\
\hline Non-Walkerton animal, not CC ST-21 (C. coli) & 8 & 0 & 0 & $8(100 \%)$ \\
\hline Total & 193 & 46 & 132 & 15 \\
\hline
\end{tabular}


Table 5 Detection of two PCR products from the pTet plasmid in Walkerton outbreak investigation strains

\begin{tabular}{|c|c|c|c|c|c|}
\hline \multirow[t]{2}{*}{ Isolate characterization } & \multicolumn{5}{|c|}{ Detection of virulence plasmid PCR products } \\
\hline & Total isolates & Both PCR products & cpp50tetO & virB4rcvap & No plasmid \\
\hline Walkerton human, CC ST-21 & 110 & $16(15 \%)$ & 0 & $3(3 \%)$ & 91 (83\%) \\
\hline Walkerton human, not CC ST-21 & 14 & $1(7 \%)$ & $2(14 \%)$ & $2(14 \%)$ & $9(64 \%)$ \\
\hline Walkerton bovine, CC ST-21 & 22 & $14(64 \%)$ & 0 & $3(14 \%)$ & $5(23 \%)$ \\
\hline Walkerton bovine, not CC ST-21 & 20 & $2(10 \%)$ & 0 & $1(5 \%)$ & $17(85 \%)$ \\
\hline Non-Walkerton human, CC ST-21 & 10 & $9(90 \%)$ & 0 & $1(10 \%)$ & 0 \\
\hline Non-Walkerton human, not CC ST-21 & 4 & 0 & 0 & 0 & $4(100 \%)$ \\
\hline Non-Walkerton animal, CC ST-21 & 5 & $2(40 \%)$ & 0 & 0 & $3(60 \%)$ \\
\hline Non-Walkerton animal, non CC ST-21 & 8 & $4(50 \%)$ & 0 & 0 & $4(50 \%)$ \\
\hline \multicolumn{6}{|l|}{ C. coli } \\
\hline Total & 193 & 48 & 2 & 10 & 133 \\
\hline
\end{tabular}

(Table 3). Sanger sequencing and BLAST analysis of this 3 -kb product indicated that the amplified sequence was consistent with a $\sim 388 \mathrm{~kb}$ inversion between the tlp3 and tlp12 genes present in the four sequenced outbreak isolates. A third possible inversion of $297 \mathrm{~kb}$ located between repeats 1 and 3 (in the two tlp3 genes) was not detected in this study. The sizes of the 388-kb inversion and potential 296-kb inversion would each be increased by $38 \mathrm{~kb}$ in isolate $00-2544$ due to the insertion of the CJIE1 prophage (see Fig. 1).

\section{Discussion and Conclusions}

The four outbreak isolates characterized in this work had closed, finished genomes with almost identical gene content, with the exception of a deletion of the CJIE1 prophage, four additional tRNA loci in isolate 00-2426, and a hqSNV inactivating the threonine/serine transporter SstT. They also had the same homopolymeric tract content and location, minimal hqSNV variability, and limited cgMLST allelic variation, especially in the context of a larger population of ST21 strains. These data provided strong support for the conclusions presented in earlier work that the strain-specific differences in cell culture adherence and invasion assays [28] and protein expression [29] resulted from the presence or absence of the CJIE1 prophage. Though artifacts may arise from the use of SNV analysis with C. jejuni [16], we found that both SNV analysis and cgMLST delivered congruent results in that they clustered the four outbreak isolates closely together within the $C$. jejuni phylogenetic structure. Both hqSNV analysis and cgMLST demonstrated consistent differences between isolates that were due to allelic differences at a small number of loci, supporting the hypothesis that they were changing rapidly as part of a freely recombining population. Interpretation of whole genome sequence data relies on large datasets to allow conclusions about phylogenetic relationships to be drawn; a larger dataset was used for the cgMLST analysis, which enabled a much more detailed picture of phylogenetic relationships. Crucial phylogenetic information not available using hqSNV analysis or cgMLST was provided by comparing the complete genomes of WGS strains, which contain the accessory genome for each strain, using whole genome NeighborJoining phylogenetic methods.

\section{Homopolymeric tracts are useful for assessing relationships among isolates}

The locations of homopolymeric tracts were identical in the four outbreak strains and variable from other strains assessed. These observations were consistent with the phylogenetic relationships among isolates determined in this work showing that the four Walkerton outbreak strain 1 isolates were related to, but distinct from, strain NCTC11168 (Fig. 3, Additional file 2: Figure S1). They also support our conclusions that 00-2425, 00-2426, 00-2538, and 00-2544 are genetically almost identical and are most certainly clonally derived, thus highlighting the value of using comparisons of homopolymeric tract locations as an adjunct method to confirm phylogenetic relationships within $C$. jejuni. While the homopolymeric tract content and location may provide supporting data regarding the relatedness of strains for outbreak analysis, it seems unlikely that these data will supplant cgMLST and SNV analysis for outbreak detection and analysis or investigations into population genetics of the organism.

Changes in homopolymeric tract length affect expression of the genes with which they are associated and their association with different loci in different strains suggests that these homopolymeric tracts contribute to the adaptation of strains through selection in different environments or niches [33]. When population data are available, each of the homopolymeric tract length variants detected is best described as a proportion or percentage of the total population [37-39], and even individual strains of $C$. jejuni are a heterogeneous population of organisms capable of generating multiple 
phenotypes through adaptation to external or environmental conditions [40]. However, due to the high rate of mutation causing nucleotide changes in homopolymeric tracts [33] SNVs arising from changes in polyG/polyC tract lengths are not useful for phylogenetic or molecular epidemiologic analysis and it may be of merit to consider SNV differences after removing them from the analysis [17], as well as adjusting the lengths of the homopolymeric tracts to encode the full length protein when submitting sequence data to the NCBI.

\section{Potential significance of large chromosomal inversions}

Detection of large chromosomal inversions between chemotaxis proteins/Tlps containing large conserved repeats indicates that $C$. jejuni genomes may be more dynamic than previously appreciated and suggests that this property may have adaptive consequences. Chromosomal inversions may not be uncommon in $C$. jejuni and related organisms. In Helicobacter pylori, an organism closely related to Campylobacter spp., large chromosomal inversions were associated with insertion element IS605 (producing a $75 \mathrm{~kb}$ inversion) or inverted copies of repeat 7 (producing an $83 \mathrm{~kb}$ inversion) [41]. In C. jejuni strains, large genomic rearrangements induced by lytic bacteriophage predation resulted from recombination between two copies of the CJIE1 Mu-like bacteriophage [42]. One inversion characterized in this study was centered on the origin of replication and had measurable biological consequences that included increased survival in adverse ecological conditions. Inversions between $r r n$ operons in the genomes of specialist Salmonella enterica serotypes Typhi, Paratyphi C, Gallinarum, and Pullorum have been detected at much higher rates than in the generalist $S$. Typhimurium in spite of similar frequencies of inversion, an observation attributed to decreased survival of $S$. Typhimurium due to selection pressures inherent to its generalist lifestyle [43]. C. jejuni associated with several sequence types, including ST21, have been described as generalists on the basis of phenotypic flexibility and high genetic microdiversity [44]. It is not clear whether this affects the frequency of chromosomal inversions in this organism, or whether there are chromosomal inversions mediated by rrn operons or other repeats detected in $C$. jejuni chromosomes. Inversions that do not maintain the genome balance, defined as the lengths of replichores between the origin and terminus of replication, are also subject to selective pressure [21]. The $93 \mathrm{~kb}$ inversion we detected would likely not affect the genome balance except in the case of strain 00-2544. Further investigations are necessary to determine whether the three repeats found in strains used in this study are restricted to only a subset of $C$. jejuni or are more generalized throughout the population, as well as whether the gene content of the 93$\mathrm{kb}$ invertible region is relatively conserved or diverse in content. Too few data were obtained to enable determination of an estimate of inversion frequencies between the repeats. It has been suggested previously that repeats separated by sequence comprising more than $10 \%$ of the chromosome length are rare, indicating strong counter selection against very large inversions [45]. Higher rates of inversion would tend to homogenize a population distinguishable by other means, while lower rates may lead to increased temporal and geographic stability, thus enabling inversion status as a useful measure for population biology. This contention is supported by the results of experiments assessing the frequency of the $93 \mathrm{~kb}$ inversion in Walkerton outbreak isolates.

Differences in gene placement between the leading or lagging strand and differences in distance from the origin of replication that would result from inversion of the genomic segment(s), with possible concomitant gene dosage changes, may be associated with differences in gene and protein expression that would favor different lifestyles or environments. However, since the 93-kb inverted element is relatively small and close to the origin of replication there may not be sufficient difference in gene dosage to be of significance. This is a subject for future investigation.

Analysis of complete, finished whole genome sequences of four outbreak isolates revealed chromosomal inversions and provided insight into the capability of using changes in homopolymeric tract locations as an estimator of isolate relatedness. The examination of whole genome sequences for properties in addition to those that assess phylogenetic relatedness for resolving outbreaks provides additional valuable insights into the biology of the organism. By closing and finishing a representative subset of outbreak genomes, we have been able to gain insight into the dynamics of the Campylobacter genome, information that would not have been reliable or accurate using draft genomes alone. This analysis is anticipated to spur additional research into unravelling the intricacies of the Campylobacter genome and augment our existing knowledge of outbreakrelevant genomic markers.

\section{Methods}

\section{Strains and growth conditions}

The four isolates selected for whole genome sequencing and comparative genomic analysis were associated with the investigation into the spring 2000 Campylobacter and E. coli outbreak in Walkerton, Ontario, Canada, and have been described previously (see Background). Other isolates were included to determine the prevalence of a $93 \mathrm{~kb}$ inversion and a large 46,902 bp pTet plasmid in isolates associated with the Walkerton outbreak investigation. These isolates were all linked to the Walkerton outbreak or the subsequent investigation $[26,45]$ and 
included C. jejuni and C. coli isolates not previously characterized by PFGE or other molecular methods. Among the isolates used were 124 isolates from humans (110 outbreak types and 14 non-outbreak types) and 43 cattle feces isolates (23 outbreak types and 20 nonoutbreak types) collected as part of the outbreak investigation. Additional C. jejuni non-outbreak isolates were analyzed to provide additional context for the outbreak [26]: 13 non-outbreak C. jejuni from five different Canadian provinces and one from Egypt obtained from 2000 to 2003 (11 with outbreak type CC ST-21 but different PFGE types than outbreak isolates); two non-outbreak $C$. jejuni from Ontario cattle isolated in 2000; one Ontario canine isolate from 2000 with CC ST-21 but different PFGE types than outbreak strains; two Louisiana chicken isolates from 1999 with CC ST-21. Eight C. coli isolates obtained during the outbreak investigation, two from humans and six from cattle stools were also used here. MLST was done as described previously [26].

C. jejuni isolates were maintained in either $20 \%$ skim milk or glycerol peptone water ( $25 \% \mathrm{v} / \mathrm{v}$ glycerol, $10 \mathrm{~g} / \mathrm{L}$ neopeptone, $5 \mathrm{~g} / \mathrm{L} \mathrm{NaCl}$ ) at $-80{ }^{\circ} \mathrm{C}$. For use, $C$. jejuni isolates with a low passage number were retrieved from storage at $-80{ }^{\circ} \mathrm{C}$, plated to Oxoid Mueller-Hinton agar (Oxoid Inc.) containing 10\% sheep red blood cells (OMHA + blood), and grown for $48-72 \mathrm{~h}$ at $37{ }^{\circ} \mathrm{C}$ under a microaerobic atmosphere $\left(5 \% \mathrm{O}_{2}, 10 \% \mathrm{CO}_{2}\right.$, $85 \% \mathrm{~N}_{2}$ ).

PCR for determining the presence of the 93- and 388-kb genomic inversions and pTet plasmids

All PCR reactions were done using DNA extracted from bacteria using a Gentra Systems PUREGENE DNA Isolation kit (Qiagen) according to the instructions of the manufacturer. PCR reactions were run using reagents from FastStart Taq DNA Polymerase kits (Roche). PCR reactions using the $\mathrm{Cj}$ primer sets to assess the presence or absence of the $\sim 93-\mathrm{kb}$ and $\sim 388-\mathrm{kb}$ inversions (Table 1) each consisted of a $50 \mu \mathrm{l}$ reaction mix at final $\mathrm{MgCl}_{2}$ concentrations of $2.0 \mathrm{mM}, 0.2 \mathrm{mM}$ of each dNTP, $0.2 \mu \mathrm{M}$ of each primer, and $0.2 \mathrm{U}$ of FastStart DNA polymerase. PCR reactions to detect the 93-kb inversion were run using $\mathrm{Cj}-\mathrm{F} 1$ and $\mathrm{Cj}-\mathrm{F} 2$ primers, while the noninverted configuration was detected using the $\mathrm{Cj}-\mathrm{F} 1$ and $\mathrm{Cj}-\mathrm{R} 1$ primer set. Amplification for both the $\mathrm{Cj}-\mathrm{F} 1 / \mathrm{Cj}-$ $\mathrm{R} 1$ and $\mathrm{CjF} 1 / \mathrm{CjF} 2$ sets of primers utilized the following cycles: 1 cycle of $95{ }^{\circ} \mathrm{C}$ for $2 \mathrm{~min} ; 35$ cycles of $94{ }^{\circ} \mathrm{C}$ for $30 \mathrm{~s}, 56{ }^{\circ} \mathrm{C}$ for $30 \mathrm{~s}, 68^{\circ} \mathrm{C}$ for $5 \mathrm{~min} ; 1$ cycle for final extension at $72{ }^{\circ} \mathrm{C}$ for $7 \mathrm{~min} ; 4{ }^{\circ} \mathrm{C}$ until samples were retrieved. The amplicon obtained using both the F1-R1 primers and F1-F2 primers was approximately $2.5 \mathrm{~kb}$ in size. Strain RM1221 was the positive control for the non-inverted configuration, NCTC11168 was the positive control for the inversion, and a tube or well containing water instead of DNA was a negative control to detect inappropriate amplification. For detection of the 388-kb inversion the Cjinv5 primers cycle conditions were: 1 cycle of $95{ }^{\circ} \mathrm{C}$ for $2 \mathrm{~min} ; 35$ cycles of $94{ }^{\circ} \mathrm{C}$ for $30 \mathrm{~s}, 47.8{ }^{\circ} \mathrm{C}$ for $30 \mathrm{~s}, 68{ }^{\circ} \mathrm{C}$ for $4 \mathrm{~min} ; 1$ cycle for final extension at $72{ }^{\circ} \mathrm{C}$ for $7 \mathrm{~min} ; 4^{\circ} \mathrm{C}$ until samples were retrieved. The Cjinv5 amplicon was approximately $2.5 \mathrm{~kb}$. Other potential inversion ends were tested but no amplicons were obtained; the primers used were therefore not included here.

Two sets of primers were designed for detection of the pTet plasmid in C. jejuni isolates associated with the Walkerton outbreak investigation using the sequence of the plasmid obtained for C. jejuni strain 00-2544 (Table 1). Each $50 \mu \mathrm{l}$ reaction mix contained final $\mathrm{MgCl}_{2}$ concentrations of $2.0 \mathrm{mM}, 0.2 \mathrm{mM}$ of each dNTP, 0.5 $\mu \mathrm{M}$ of each primer, and $0.2 \mathrm{U}$ of FastStart DNA polymerase. Amplification cycles were the same as above for the primers to detect inverted or non-inverted genomic sequences, but the cpp50tetO primer set used an annealing temperature of $53{ }^{\circ} \mathrm{C}$ and an extension time of $45 \mathrm{~s}$ while the virB4rcvap primer set had an annealing temperature of $50{ }^{\circ} \mathrm{C}$ and a 2 min extension time. The product of the cpp50tetO amplification was $1018 \mathrm{bp}$; that of the virB4rcvap amplification was $1410 \mathrm{bp}$. These two primer sets amplified regions that were approximately $19 \mathrm{~kb}$ apart in the $46 \mathrm{~kb}$ plasmid. The positive control was DNA from 00-2544, the negative control was DNA from NCTC11168, and a water blank was included as a contamination control for each run.

All PCR reactions were performed using a Gene Amp 9700 thermocycler (Applied Biosystems). Visualization of PCR products was accomplished using submarine gel electrophoresis followed by staining with GelRed Nucleic Acid Stain (Cedarlane) or analysis using a Qiaxcel instrument (Qiagen).

\section{Genome sequencing, assembly, closure, and annotation Sequencing}

Genomic DNA was prepared from four selected Walkerton outbreak isolates cultured overnight at $42{ }^{\circ} \mathrm{C}$ on OMHA + blood using Epicentre Metagenomic DNA Isolation kits for Water (Illumina) according to the manufacturer's instructions. Quantitation of DNA was accomplished using Qubit dsDNA BR assay kits (Life Technologies, Invitrogen). Sample libraries were prepared using MiSeq Nextera ${ }^{\bullet}$ XT DNA library preparation kit (Illumina). Whole genome sequencing was performed by 250 bp paired-end read sequencing on the Illumina MiSeq sequencer using MiSeq ${ }^{\bullet}$ Reagent Kit V2 and 500 cycles on the Illumina MiSeq platform to obtain an average genome coverage of 30-50×. Sequence reads were assembled into contigs using the SPAdes assembler (v3.0 [46]). Contigs smaller than 1-kb and with average 
genome coverage less than $15 \times$ were filtered and removed from the analysis. The remaining contigs were closed and finished using Staden gap v4.10 by read mapping to the reference genome, NCTC11168, and a combination of PCR and Sanger sequencing for gap closure. Fasta files for each genome were sent to Genomes (NCBI) for annotation using the NCBI prokaryotic annotation pipeline. Additional information for selected loci was then added manually.

\section{Core genome hqSNV analysis}

A core genome phylogeny was constructed from high quality variants within the core genome using the read data for C. jejuni isolates 00-2425, 00-2426, 00-2538, 00-2544, and 29 related isolates from GenBank using the closed and finished genome of isolate $00-2425$ as the reference. The Illumina MiSeq data for the isolates sequenced in this study were first concatenated into one fastq file per isolate containing both forward and reverse reads. Fasta files for closed, finished genomes of 29 additional isolates were downloaded from GenBank and converted to reads of $250 \mathrm{bp}$ using custom Perl scripts (WombacShred [47]) to generate fastq files. The set of reads generated for each isolate was used for phylogenetic SNV analysis using the PHAC-NML high-quality single nucleotide variants (SNVPhyl) pipeline [48].

In summary, read data for each genome was mapped against a reference closed and finished genome $($ NCTC11168 = ATCC 700819) using SMALT (v. 0.7.5; Wellcome Trust Sanger Institute, Cambridge, UK). The SMALT parameters used were a smalt index kmer size of 13 , a step size of 6 , and a minimum alignment fraction of 0.5. Variants were called using FreeBayes (v. 0.9.20 $[49,50])$ with the "-ploidy 1" parameter for haploid variant calling. For each VCF file created by FreeBayes, an in-house script was used to filter out complex variant calls with indels, split the remaining complex variant calls into single variant calls, and create new VCF files. The BCFtools component of the SAMtools package (v. 1.3 [51]) was used as a second variant caller to validate the variant calls made by FreeBayes. All variant and non-variant calls from FreeBayes and BCFtools were merged together and positions where variant calls were not in agreement between both variant callers and which did not have a minimum coverage of 10 , minimum alternative ratio of 0.75 , and minimum mean mapping quality of alternative alleles of 30 were excluded from the analysis. Additional filtering was performed to remove variant calls within repetitive regions (identified using MUMmer v3.23 [31] with a minimum percent identity of 90 and minimum length of $150 \mathrm{bp}$ ) and those within high SNV-density regions (identified with a sliding window of size $20 \mathrm{bp}$ and minimum threshold of two SNVs within this window size) to be removed. All remaining variant calls were merged into a single metaalignment file. The meta-alignment was used to generate a maximum likelihood phylogenetic tree with PhyML [52]. The dendrogram was depicted with FigTree v1.4 [53]. Metadata for isolates obtained from the NCBI repositories was obtained from the scientific literature. When necessary, MLST designations were obtained from WGS data using custom in-house scripts [54].

\section{Pan-genome BLAST Atlas}

A pan-genome BLAST Atlas was created using GView [55] and GView Server [56]. The pan-genome was constructed iteratively by starting with the predicted chromosomal regions (CDS) in 00-2425 and concatenating unique regions among the other three genomes using MUMMer (v3.1) alignments. Next BLASTn was performed between the pan-genome and each of the other genomes. Regions on each genome reporting a BLAST hit above the threshold cutoff ( $80 \%$ identity, minimum HSP length of $100 \mathrm{bp}$, and expect value of (1e-10)) were considered a valid match and drawn on the pan-genome BLAST atlas.

\section{cgMLST analysis}

A cgMLST scheme was developed as previously described [57] using allele definitions obtained from BIGSdb [32]. A dataset of genomes comprised of isolates from ST21, which included genomes obtained from BIGSdb $(n=181)$ and those sequenced by our group $(n$ =17), was analyzed by cgMLST. Analysis was performed using the Microbial In Silico Typer (MIST) software [58]. Briefly, MIST was used to query each genome for all known alleles at each locus by homology searching, with novel alleles identified and provided with a unique allele number. Loci with apparent truncations or sequencing errors in any of the genomes in the dataset were excluded from the analysis. The remaining loci $(n=732)$ were used to define the cgMLST scheme used in this study. The genetic distance between each pair of strains was calculated using the Hamming distance [59]. Hierarchical clustering was performed by the Unweighted Pair Group Method with Arithmetic Mean (UPGMA) using the hclust function in $\mathrm{R}[60]$.

\section{Additional files}

Additional file 1: Figure S1. Dendrogram showing Neighbour-Joining analysis of $C$. jejuni complete whole genomes. The alignment was performed using Progressive Mauve [61] and the dendrogram produced using FigTree V1.4 [53]. (PDF 3 kb)

Additional file 2: Table S1. Selected SNVs detected using NUCmer for comparing all outbreak isolates using 00-2425 as reference. (DOCX $20 \mathrm{~kb}$ )

Additional file 3: Table S2. Homopolymeric G/C tracts eight nucleotides or longer in selected whole genome-sequenced C. jejuni. (DOCX $27 \mathrm{~kb}$ ) 
Additional file 4: Figure S2. Comparison of the $~ 93-k b$ invertible region for strains NCTC11168 = ATCC 700819 and 00-2425. Note that the 00-2425 region has been inverted for the diagram to highlight the synteny of this region between the two strains. The genes outside this region were found in the same locations in both NCTC11168= ATCC 700819 and $00-2425$, but appear at different places in the figure due to this inversion. Diagrams for each strain were obtained using GView Server [56] and further annotated using Adobe Illustrator. (PDF $177 \mathrm{~kb}$ )

Additional file 5: Table S3. Comparison of the $\sim 93-k b$ inverted region of C. jejuni strains 00-2425 and NCTC11168. (DOCX $26 \mathrm{~kb}$ )

Additional file 6: Figure S3 Repeat sequences associated with isolates 00-2425, 00-2426, 00-2538, and 00-2544. (DOCX 22 kb)

Additional file 7: Figure S4. Isolate 00-2425 alignments of repeats associated with chemotaxis proteins. (DOCX $25 \mathrm{~kb}$ )

\section{Abbreviations}

BIGSdb: Bacterial Isolate Genome Sequence Database; CC: Clonal complex; cgMLST: Core genome multilocus sequence typing; flaSVR: Flagellin A locus short variable region; HL: Heat-labile (serotype); hqSNV: High quality single nucleotide variant; HS: Heat-stable (serotype); MLST: Multilocus sequence typing; MOMP: Major outer membrane protein; nt: Nucleotide; PFGE: Pulsed-field gel electrophoresis; porA gene (MOMP): porA gene encoding the C. jejuni major outer membrane protein; rMLST: Ribosomal MLST; ST: C. jejuni MLST sequence type; Tlp: Transducer-like protein; WGS: Next-generation whole-genome sequencing

\section{Acknowledgements}

Miles Majcher and Jason Moses performed the MALDI-TOF analysis to recheck the species identity of several Campylobacter isolates. Phil Mabon provided support for bioinformatics analysis and editing of bioinformatics methodology in the Methods section. Genome sequencing was done with the aid of the Genomics Core Facility at the National Microbiology Laboratory, Winnipeg. This work would not have been possible without the close collaboration and support of the Public Health Laboratory of Ontario, Public Health Laboratory of Alberta, Laboratoire de santé publique du Quebec, (LSPQ; Québec Public Health Laboratory), and public health authorities in New Brunswick.

\section{Funding}

Funding for this work came from Public Health Agency of Canada A-base funding and through the Government of Canada's Genomics Research and Development Initiative.

\section{Availability of data and materials}

GenBank Accession numbers. The closed, finished genomes have been deposited in GenBank under the following accession numbers: 00-2425, NC_022362.2 and CP006729.2; 00-2426, NC_022352.2 and CP006708.2; 002538, NC_022351.2 and CP006707.2; 00-2544, NC_022353.2 and CP006709.2. The sequence of the plasmid from isolate 00-2544 has been deposited in GenBank with the accession number NC_022354.1.

WGS sequence data submitted to the Sequence Read Archive (SRA) at NCBI. Sequence reads for nine isolates were submitted under BioProject accession number PRJNA350537 (study SRP092105). The SRA accession number and BioSample accession number for each isolates are: 07-3238, SRR4451710, SAMN05942199: 07-4228, SRR4451709, SAMN05942200; 075581, SRR4451708, SAMN05942201; 07-5583, SRR4451707, SAMN05942202; CCGYHR028, SRR4451714, SAMN05942203; CI 0987, SRR4451713, SAMN05942204; CI_5328, SRR4451712, SAMN05942205; CI_5357, SRR4451711, SAMN05942206; CI_5429, SRR4451706, SAMN05942207.

Otherwise, the datasets supporting the conclusions of this article are included within the article and its additional files.

\section{Author's contributions}

CGC, ENT, and MW designed the experiments; AR provided human and material resources for the work and reviewed the manuscript; MW sequenced and closed the genomes; CGC and MW analyzed and annotated the genomes; CB, MW, and CGC performed the hqSNV analysis; ENT and DB performed cgMLST using a C. jejuni ST21 database; CGC and CG performed PCR for chromosomal inversions and plasmid genes; CB analyzed the content of the $93 \mathrm{~kb}$ invertible segment, obtained COG data, and prepared the figure; CGC, ENT, and CB interpreted the data, wrote, and edited the manuscript. AP provided bioinformatics support, wrote or edited sections of the manuscript associated with bioinformatics tools, and edited the manuscript. All authors read and approved the final manuscript.

\section{Author's information}

Not applicable.

\section{Competing interests}

The authors declare that they have no competing interests.

\section{Consent for publication}

Not applicable.

\section{Ethics approval and consent to participate}

Not applicable.

\section{Author details}

${ }^{1}$ Division of Enteric Diseases, National Microbiology Laboratory, Public Health Agency of Canada, Canadian Science Centre for Human and Animal Health 1015 Arlington Street, Winnipeg, MB R3E 3R2, Canada. ${ }^{2}$ Bioinformatics Core Facility, National Microbiology Laboratory, Public Health Agency of Canada, Winnipeg, MB R3E 3R2, Canada. ${ }^{3}$ Division of Enteric Diseases, National Microbiology Laboratory, Public Health Agency of Canada, Lethbridge, $A B$ T1J 3Z4, Canada. ${ }^{4}$ Department of Biological Sciences, University of Lethbridge, Lethbridge, $A B$, Canada.

Received: 19 July 2016 Accepted: 23 November 2016 Published online: 03 December 2016

\section{References}

1. Coker AO, Isokpehi RD, Thomas BN, Amisu KO, Obi CL. Human campylobacteriosis in developing countries. Emerg Infect Dis. 2002:8:237-43.

2. Kirkpatrick BD, Tribble DR. Update on human Campylobacter jejuni infections. Curr Opin Gastroenterol. 2010;27:1-7.

3. Newell DG, Coopmans M, Verhoef L, Duizer E, Aidara-Kane A, Sprong H, et al. Food-borne diseases - the challenges of 20 years ago still persist while new ones continue to emerge. Int J Food Microbiol. 2010;139:S3-S15.

4. Silva J, Leite D, Fernandes M, Mena C, Gibbs PA, Teixeria P. Campylobacter spp. as a foodborne pathogen: a review. Frontiers Microbiol. 2011;2:200.

5. Thomas MK, Murray R, Flockhart L, Pintar K, Pollari F, Fazil A, et al. Estimates of the burden of foodborne illness in Canada for 30 specified pathogens and unspecified agents, circa 2006. Foodborne Path Dis. 2013;10:639-48.

6. Bronowski C, James CE, Winstanley C. Role of environmental survival in transmission of Campylobacter jejuni. FEMS Microbiol Lett. 2014;365:8-19.

7. Moore JE, Corcoran D, Dooley JSG, Fanning S, Lucey B, Matsuda M, et al. Campylobacter. Vet Res. 2005:36:351-82.

8. Ahmed MU, Dunn L, Ivanova EP. Evaluation of current molecular approaches for genotyping Campylobacter jejuni strains. Foodborne Path Dis. 2012;9:375-85.

9. Taboada EN, Clark C, Sproston EL, Carrillo CD. Current methods for molecular typing of Campylobacter species. J Microb Meth. 2013;95:24-31.

10. Frost JA. Current epidemiological issues in human campylobacteriosis. J Appl Micriobiol. 2001;90:85S-95S.

11. Gilmour MW, Graham M, Reimer A, van Domselaar G. Public health genomics and the new molecular epidemiology of bacterial pathogens. Public Health Genomics. 2013:16:25-30.

12. Hasman H, Saputra D, Sicheritz-Pontin T, Lund O, Svendsen CA, FrimodtMøller $\mathrm{M}$, et al. Rapid whole-genome sequencing for detection and characterization of microorganisms directly from clinical samples. J Clin Microbiol. 2014:52:139-46.

13. Robinson ER, Walker TM, Pallen MJ. Genomics and outbreak investigation: from sequence to consequence. Genome Med. 2013;5:36.

14. Wilson DJ. Insights from genomics into bacterial pathogen populations. PLoS Pathog. 2012;8, e1002874.

15. Taboada EN, Ross SL, Mutschall SK, Mackinnon JM, Roberts MJ, Buchanan CJ et al. Development and validation of a comparative genomic fingerprinting method for high- resolution genotyping of Campylobacter jejuni. J Clin Microbiol. 2012;50:788-97.

16. Méric G, Yahara K, Mageiros L, Pascoe B, Maiden MCJ, Jolley KA, et al. A reference pan-genome approach to comparative bacterial genomics: identification of novel epidemiological markers in pathogenic Campylobacter. PLoS One. 2014;9, e92798. 
17. Revez J, Zhang J, Schott T, Kivistö R, Rossi M, Hänninen M-L. Genomic variation between Campylobacter jejuni isolates associated with milk-bornedisease outbreaks. J Clin Microbiol. 2014;52:2782-6.

18. Revez J, Llarene A-K, Schott T, Kuusi M, Hakkinen M, Kivistö R, et al. Genome analysis of Campylobacter jejuni strains from a waterborne outbreak. BMC Genomics. 2014;15:768.

19. Kovanen SM, Kivistö Rl, Rossi M, Schott T, Kärkkäinen U-M, Tuuminen T, et al. Multilocus sequence typing (MLST) and whole-genome MLST of Campylobacter jejuni isolates from human infections in three districts during a seasonal peak in Finland. J Clin Microbiol. 2014;52:4147-54.

20. Cody AJ, McCarthy ND, van Rensburg MJ, Isinkaye T, Bentley SD, Parkhill J, et al. Real-time genomic epidemiological evaluation of human Campylobacter isolates by use of whole-genome multilocus sequence typing. J Clin Microbiol. 2013;51:2526-34.

21. Achaz G, Coissac E, Netter P, Rocha EPC. Associations between inverted repeats and the structural evolution of bacterial genomes. Genetics. 2003; 164:1279-89.

22. Fouts DE, Mongodin EF, Mandrell RE, Miller WG, Rasko DA, Ravel J, et al. Major structural differences and novel potential virulence mechanisms from the genomes of multiple Campylobacter species. PLoS Biol. 2005;3(1), e15.

23. Morley L, McNally A, Paszkiewicz K, Corander J, Méric G, Sheppard SK, et al. Gene loss and lineage-specific restriction-modification systems associated with niche differentiation in the Campylobacter jejuni sequence type 403 clonal complex. Appl Environ Microbiol. 2015;81:3641-47.

24. Relman DA. Microbial genomics and infectious disease. New Engl J Med. 2011:365:347-57.

25. Bruce-Grey-Owen Sound Health Unit. Waterborne outbreak of gastroenteritis associated with a contaminated municipal water supply, Walkerton, Ontario, May-June 2000. Can Comm Dis Rep. 2000;26(20):170-73.

26. Clark CG, Bryden L, Cuff W, Johnson PL, Jamieson F, Ciebin B, et al. Use of the Oxford multilocus sequence typing protocol and sequencing of the flagellin short variable region to characterize isolates from a large outbreak of waterborne Campylobacter sp. strains in Walkerton, Ontario, Canada. J Clin Microbiol. 2005:43:2080-91.

27. Barton C, Ng L-K, Tyler SD, Clark CG. Temperate bacteriophages affect pulsed-field gel electrophoresis patterns of Campylobacter jejuni. J Clin Microbiol. 2007:45:386-91.

28. Clark CG, Grant CCR, Pollari F, Marshall B, Moses J, Tracz DM, et al. Effects of the Campylobacter jejuni CJIE1 prophage homologs on adherence and invasion in culture, patient symptoms, and source of infection. BMC Microbiol. 2012;12:269.

29. Clark CG, Chong PM, McCorrister SJ, Simon P, Walker M, Lee DM, et al. The CJIE1 prophage of Campylobacter jejuni affects protein expression in growth media with and without bile salts. BMC Microbiol. 2014;14:70.

30. Kurtz S, Phillipy A, Delcher AL, Smoot M, Shumway M, Antonescu C, et al. Versatile and open software for comparing large genomes. Genome Biol. 2004;5:R12.

31. The MUMmer 3 Manual. http://mummer.sourceforge.net/manual/.

32. Jolley KA, Maiden MCJ. BIGSdb: Scalable analysis of bacterial genome variation at the population level. BMC Bioinformatics. 2010;11:595.

33. Aidley J, Bayliss CD. Repetitive DNA: a major source of genetic diversity in Campylobacter populations? In: Sheppard SK, Méric G, editors. Campylobacter ecology and evolution. Norfolk: Caister Academic Press; 2014. p. 55-72.

34. He Y, Yan X, Reed S, Xie Y, Chen CY, Irwin P. Complete genome sequence of Campylobacter jejuni YH001 from beef liver, which contains a novel plasmid. Genome Announc. 2015;3(1):e01492-14. doi:10.1128/genomeA.01492-14.

35. Marchant J, Wren B, Ketley J. Exploiting genome sequence: predictions for mechanisms of Campylobacter chemotaxis. TRENDS Microbiol. 2002;10:155-9.

36. Clark CG, Price L, Ahmed R, Woodward DL, Melito PL, Rodgers FG, et al. Characterization of waterborne outbreak-associated Campylobacter jejuni, Walkerton, Ontario. Emerg Infect Dis. 2003;9:1232-41.

37. Thomas DK, Lone AG, Selinger LB, Taboada EN, Uwiera RRE, Abbott DW, et al. Comparative variation within the genome of Campylobacter jejuni NCTC 11168 in human and murine hosts. PLoS One. 2014;9(2), e88229.

38. Jerome JP, Bell JA, Plovanich-Jones AE, Barrick JE, Brown CT, Mansfield LS. Standing genetic variation in contingency loci drives the rapid adaptation of Campylobacter jejuni to a novel host. PLoS One. 2011;6, e16399.

39. Kim J-S, Artymovich KA, Hall DF, Smith EJ, Fulton R, Bell J, et al. Passage of Campylobacter jejuni through the chicken reservoir or mice promotes phase variation in contingency genes Cj0045 and Cj0170 that strongly associates with colonization and disease in a mouse model. Microbiol. 2012;158:1304-16.
40. Revez J, Zhang J, Schott T, Llarina A-K, Rossi M, Hänninen M-L. Genetic heterogeneity of Campylobacter jejuni NCTC 11168 upon human infection. Infect Genet Evol. 2013;16:305-09.

41. Alm RA, Ling L-SL, Moir DT, King BL, Brown ED, Doig PC, et al. Genomicsequence comparison of two unrelated isolates of the human gastric pathogen Helicobacter pylori. Nature. 1999;397:176-80.

42. Scott AE, Timms AR, Connerton PL, Loc Carrillo C, Radzum KA, Connerton IF. Genome dynamics of Campylobacter jejuni in response to bacteriophage predation. PLoS Pathog. 2007;3, e119.

43. Kothapalli S, Nair S, Alokam S, Pang T, Khakhria R, Woodward D, et al. Diversity of genome structure in Salmonella enterica serovar Typhi populations. J Bacteriol. 2005;187:2638-50.

44. Gripp E, Hlahla D, Didelot X, Kops F, Maurischat S, Tedin K, et al. Closely related Campylobacter jejuni strains from different sources reveal a generalist rather than a specialist lifestyle. BMC Genomics. 2011:12:584.

45. Rocha EPC, Danchin A, Viari A. Functional and evolutionary roles of long repeats in prokaryotes. Res Microbiol. 1999:150:725-33.

46. Bankevich A, Nurk S, Antipov D, Gurevich AA, Dvorkin M, Kulikov AS, et al. SPAdes: a new genome assembly algorithm and its application to singlecell sequencing. J Comput Biol. 2012;19:455-77.

47. WombacShred. http://www.vicbioinformatics.com/software.wombac.shtml

48. PHAC-NML SNVPhyl (hqSNV) pipeline. https://github.com/phac-nml/ snvphyl-galaxy

49. FreeBayes. https://github.com/ekg/freebayes.

50. Garrison E, Marth G. Haplotype-based variant detection from short-read sequencing. arXiv preprint arXiv 2012;1207.3907 [q-bio.GN]

51. Li H, Handsaker B, Wysoker A, Fennel T, Buan J, Homer N, et al. The sequence alignment/map (SAM) format and SAMtools. Bioinformatics. 2012; 25:27-9.

52. Guindon S, Dufayard JF, Lefort V, Anisimova N, Hordijk W, Gascuel O. New algorithms and methods to estimate Maximum-Likelihood phylogenies: assessing the performance of PhyML 3.0. System Biol. 2010;59:307-21.

53. FigTree v1.4. http://tree.bio.ed.ac.uk/software/figtree/.

54. Inouye M, Dashnow H, Raven L-A, Schultz MB, Pope BJ, Tomita T, et al. Rapid genomic surveillance for public health and hospital microbiology labs. Genome Med. 2014;6:90.

55. Petkau A, Stuart-Edwards M, Stothard P, Van Domselaar G. Interactive microbial visualization with GView. Bioinformatics. 2010;26:3125-26.

56. GView Server. https://server.gview.ca. Accessed 23 Mar 2016.

57. Sheppard SK, Jolley KA, Maiden MCJ. A gene-by-gene approach to bacterial population genomics: whole genome MLST of Campylobacter. Genes. 2012; 3:261-77.

58. Kruczkiewicz P, Mutschall S, Barker D, Thomas J, Van Domselaar G, Gannon VPJ, et al. MIST: a tool for rapid in silico generation of molecular data from bacterial genome sequences. Proc. Bioinforma. 2013 4th Int. Conf. Bioinforma. Models Methods Algorithms: 316-23

59. Hamming RW. Error detecting and error correcting codes. Bell Syst Tech J. 1950;29:147-60.

60. Core Team R. R: A language and environment for statistical computing. Vienna: R Foundation for Statistical Computing; 2012.

61. Darling AC, Mau B, Blattner FR, Perna MT. Mauve: multiple alignment of conserved genomes sequences with rearrangements. Genome Res. 2004;14: 1394-403. http://darlinglab.org/mauve. Accessed 11 Jan 2016.

\section{Submit your next manuscript to BioMed Central and we will help you at every step:}

- We accept pre-submission inquiries

- Our selector tool helps you to find the most relevant journal

- We provide round the clock customer support

- Convenient online submission

- Thorough peer review

- Inclusion in PubMed and all major indexing services

- Maximum visibility for your research

Submit your manuscript at www.biomedcentral.com/submit 